OPEN ACCESS

Edited by:

Yinduo Ji,

University of Minnesota Twin Cities,

United States

Reviewed by:

Maryam Dadar,

Razi Vaccine and Serum Research

Institute, Iran

Erdong Cheng,

University of Pittsburgh Cancer

Institute, United States

*Correspondence:

XiMa

maxi@cau.edu.cn

${ }^{\dagger}$ These authors have contributed equally to this work.

Received: 02 August 2017 Accepted: 12 December 2017 Published: 22 December 2017

Citation:

Liu P, Zhao J, Guo P, Lu W, Geng Z, Levesque $C L$, Johnston $L J$, Wang $C$,

Liu L, Zhang J, Ma N, Qiao S and

Ma X (2017) Dietary Corn Bran

Fermented by Bacillus subtilis MA139 Decreased Gut Cellulolytic Bacteria and Microbiota Diversity in Finishing

Pigs.

Front. Cell. Infect. Microbiol. 7:526. doi: 10.3389/fcimb.2017.00526

\section{Dietary Corn Bran Fermented by Bacillus subtilis MA139 Decreased Gut Cellulolytic Bacteria and Microbiota Diversity in Finishing Pigs}

\author{
Ping Liu ${ }^{1+}$, Jinbiao Zhao ${ }^{1+}$, Pingting Guo ${ }^{1}$, Wenqing Lu ${ }^{1}$, Zhengying Geng ${ }^{1}$, \\ Crystal L. Levesque ${ }^{2}$, Lee J. Johnston ${ }^{3}$, Chunlin Wang ${ }^{1}$, Ling Liu ${ }^{1}$, Jie Zhang ${ }^{4}$, Ning Ma ${ }^{1}$, \\ Shiyan Qiao ${ }^{1}$ and $\mathrm{Xi}^{\mathrm{Ma}}{ }^{1,5 *}$
}

'State Key Laboratory of Animal Nutrition, College of Animal Science and Technology, China Agricultural University, Beijing, China, ${ }^{2}$ Department of Animal Sciences, South Dakota State University, Brookings, SD, United States, ${ }^{3}$ Swine Nutrition and Production, West Central Research and Outreach Center, University of Minnesota, Morris, MN, United States, ${ }^{4}$ Department of Animal Husbandry and Veterinary Medicine, Beijing Vocational College of Agriculture, Beijing, China, ${ }^{5}$ Department of Internal Medicine, Department of Biochemistry, University of Texas Southwestern Medical Center, Dallas, TX, United States

Solid-state fermentation of feedstuffs by Bacillus subtilis MA139 can reduce insoluble dietary fiber content in vitro and improve growth performance in pigs. This study was conducted to investigate the effects of dietary corn bran (CB) fermented by $B$. subtilis on growth performance and gut microbiota composition in finishing pigs. A total of 60 finishing pigs were allocated to 3 dietary treatments consisting of a control (CON) diet, a 10\% CB diet, and a 10\% fermented CB (FCB) diet in a 21 d feeding trial. Growth performance and nutrient digestibility were evaluated. Fecal samples were determined for bacterial community diversity by $16 \mathrm{~S}$ rRNA gene amplicon sequencing. The dietary $\mathrm{CB}$ and FCB did not affect growth performance of finishing pigs. The digestibility of organic matter was decreased in both CB and FCB treatments compared with CON group $(P<0.05)$. The $\alpha$-diversity for bacterial community analysis of Chao 1 in FCB treatment was lower than CON treatment $(P<0.05)$. The Fibrobacteres phylum belongs to cellulolytic bacteria was isolated, and their relative abundance in CB group showed no difference between CON and FCB treatments. The abundance of Lachnospiraceae_NK4A136_group in CB treatment was higher than CON and FCB groups $(P<0.05)$, whereas the population of norank_f_Prevotellaceae was higher in FCB group compared to CON and CB groups $(P<0.05)$. In conclusion, dietary FCB decreased the abundance of bacterial communities, particularly the population of bacteria related to cellulolytic degradation.

Keywords: Bacillus subtilis, cellulolytic bacteria, corn bran, dietary fiber, fermentation, gut microbiota

\section{INTRODUCTION}

The consumption of dietary fiber plays an essential role in maintaining homeostasis of the gut ecosystem. Bacterial metabolites and prebiotics from dietary fiber are involved in various physiological processes for modulating health in humans and animals (Desai et al., 2016; Martens, 2016). Low fiber intake often results in chronic diseases related to nutrient metabolism and 
gut inflammatory disorders, such as obesity, diabetes, and inflammatory bowel diseases (Han et al., 2017; Ma et al., 2017). Thus, there is an increasing interest focused on the link between different fibrous ingredients and gut health. High fiber diets improved intestinal morphology, mucin secretion, and growth of beneficial bacteria (Hedemann et al., 2009; Chen H. et al., 2015; Tian et al., 2017). This has been attributed to the role of short-chain fatty acids (SCFAs) in the gut health and they are end-products of dietary fiber fermented by gut microbiota (Chen J. et al., 2015). The major SCFAs are acetate, propionate, and butyrate, they serve as energy substrates for the colonic epithelium and modulate the immune system (Fan et al., 2015; Koh et al., 2016). The effects of high fiber on growth performance in pigs have been studied. Some reports indicated that fiberrich diets increased weight gain (Bertram et al., 2009; Gerritsen et al., 2012), while other researchers showed reduced growth or no influence on growth performance accompanied by reduced digestibility of nutrients and energy (Jaworski et al., 2017; Morowitz et al., 2017).

The milling of corn for food constituents results in a large number of co-products. Corn bran $(\mathrm{CB})$ is a common fibrous corn by-product that has been widely utilized in animal feed due to its relatively lower cost. CB primarily consists of insoluble fiber with $\sim 280 \mathrm{~g} / \mathrm{kg}$ cellulose and $700 \mathrm{~g} / \mathrm{kg}$ hemicellulose. This indigestible corn bran contains many bioactive components, such as corn fiber gum, cellulosic fiber gel, and xylo-oligosaccharides (Rose et al., 2010). Previous studies have shown that arabinoxylan and cellulose were the major components extracted from wheat bran, which exerted positive effects on intestinal barrier function and increased the population of Lactobacillus in piglets (Chen $\mathrm{H}$. et al., 2015; Hashemi et al., 2017). In contrast, information about how $\mathrm{CB}$ impacts the gut bacterial community is very limited.

To harvest the production of useful bioactive components from corn barn, different processing technologies have been used. Fermentation technology has been applied for modification of biological materials into useful products and for reducing the insoluble fiber content of feed (Pallin et al., 2016). Bacillus subtilis MA139 was identified in previous study having high antimicrobial activity against Escherichia coli, Salmonella typhimurium, and Staphylococcus aureus (Yang et al., 2015). Diets that contained B. subtilis MA139 improved daily gain and feed conversion in weaned pigs, and $B$. subtilis was recommended as a potential alterative to in-feed antibiotics (Horita et al., 2015). B. subtilis can also produce various carbohydrase enzymes for polysaccharide degradation (Rhee et al., 2014). B. subtilis MA139 co-inoculated with lactic acid bacteria were applied with solid-state fermentation for the production of fermented wheat bran and soybean bran feedstuffs (Vijayaraghavan et al., 2016). However, the effect of dietary $\mathrm{CB}$ fermented by B. subtilis in pigs has not been reported. It is important to understand how

\footnotetext{
Abbreviations: ADFI, average daily feed intake; ADG, average daily gain; AIA, acid insoluble ash; ANOVA, one-way analysis of variance; $\mathrm{CB}$, corn bran; $\mathrm{CON}$, control; CP, crude protein; DM, dry matter; EE, ether extract; FCB, fermented corn bran; FCR, feed conversion ratio; GE, gross energy; MNB, mixed nutrient broth; MRS, de Man, rogosa and sharpe; OM, organic matter; OTUs, operational taxonomic units; SCFAs, short-chain fatty acids; TDF, total dietary fiber; YDP, yeast peptone dextrose.
}

complex polysaccharides influence gut microbiota in humans and monogastric animals. Therefore, the objective of the present study was to compare the effects of diets that contained $\mathrm{CB}$ or fermented $\mathrm{CB}$ (FCB) on growth performance, nutrient digestibility, and gut microbiota composition in finishing pigs.

\section{MATERIALS AND METHODS \\ Preparation of Fermented CB}

This study was carried out in accordance with the recommendations of "Laboratory Animals-Guideline of Welfare and Ethics of China (ICS 65.020.30), the Institutional Animal Care and Use Committee of China Agricultural University." The protocol was approved by the Institutional Animal Care and Use Committee of China Agricultural University.

Corn bran was purchased from Wellhope Agri-tech Co. Ltd. (Beijing, China). Bacillus subtilis MA139 was provided by Ministry of Agriculture Feed Industry Centre on September 5, 2016 (Beijing, China), and the Enterococcus faecium and Saccharomyces cerevisae were purchased from Yaxin Biotechnology Co. Ltd. (Taiwan, China). Bacillus subtilis MA139 and $S$. cerevisae were cultured aerobically in mixed nutrient broth $(\mathrm{MNB})$ and yeast peptone dextrose (YDP) by shaking at $250 \times$ $\mathrm{g}$ for $24 \mathrm{~h}$. E. faecium was grown in MRS (de Man, Rogosa and Sharpe) broth in $5 \% \mathrm{CO}_{2}$ incubator at $37^{\circ} \mathrm{C}$ for $22 \mathrm{~h}$ as previous report (Vijayaraghavan et al., 2016). The unsterilized CB was mixed with the starter cultures including B. subtilis MA139, E. faecium, and $S$. cerevisae in a ratio of 10:1 (w/v). After mixing, the treated CB contained $5.7 \times 10^{7} \mathrm{cfu} / \mathrm{g} \mathrm{B}$. subtilis MA139, 2.6 $\times 10^{7} \mathrm{cfu} / \mathrm{g}$ E. faecium, and $1.5 \times 10^{7} \mathrm{cfu} / \mathrm{g}$ S. cerevisae. The $\mathrm{CB}$ was packed and sealed in a polythene bag with a capacity of $25 \mathrm{~kg}$ (Rou Duoduo Biotechnology Co., Beijing, China) at $30^{\circ} \mathrm{C}$ for $14 \mathrm{~d}$. The bag was equipped with a one-way value to release gas produced during the fermentation. At the end of the solid-state fermentation, the treated CB contained 0.27 $\mathrm{mg} / \mathrm{g}$ butyrate and $0.93 \mathrm{mg} / \mathrm{g}$ lactate at $\mathrm{pH} 6.2$ analyzed by high performance ion chromatography and $\mathrm{pH}$ meter. The final moisture content of FCB was $\sim 30 \%$ according to method 930.15 (AOAC International, 2007). Samples of CB and FCB were collected for chemical analysis, including starch, crude protein, cellulose, hemicellulose, lignin, soluble dietary fiber, insoluble dietary fiber, and non-starch polysaccharide.

\section{Animals, Diet Treatments, and Sampling}

Finishing pigs $[n=60$, Duroc $\times$ (Landrance $\times$ Yorkshire), $135 \pm 1 \mathrm{~d}$ age, $65.73 \pm 0.7 \mathrm{~kg}$ body weight] were allocated randomly to 3 groups balancing for litter and gender. Pigs were housed in commercial flat-deck pens (4 pigs per pen, 5 pens per treatment). Room temperature was maintained at $22^{\circ} \mathrm{C}$, and the humidity was maintained constant at $65 \sim 75 \%$. Water and feed were provided ad libitum. Twenty pigs in each group received one of 3 experimental diets (Supplemental Table 1) based on corn and soybean meal included the control (CON) diet, a 10\% corn barn (CB) diet, and a 10\% fermented corn barn (FCB) diet. Seven days of acclimation to diets and pens were allowed before the trial started. The experiment lasted for $21 \mathrm{~d}$. 
The amount of feed provided for pigs was recorded and unconsumed feed was weighed daily for the determination of average daily feed intake (ADFI) (Jaworski et al., 2017). Pigs were weighed individually on day 0 and 21 . Average daily gain (ADG) was calculated as weight gain (final body weight-initial body weight) divided by the number of treatment days. Feed conversion ratio (FCR) was calculated as the ratio of ADFI and ADG (Jaworski et al., 2017). A sample of each of the experimental diets was collected for chemical analysis. A fecal sample ( $200 \mathrm{~g})$ was collected on d 18,19, and 20 from 1 pig per pen ( $n=5$ pigs per treatment) and pooled prior to drying. Feed and fecal samples were oven-dried at $65^{\circ} \mathrm{C}$ for $72 \mathrm{~h}$. All samples were ground to pass through 40-mesh sieve for nutrient digestibility analysis.

On $21 \mathrm{~d}$, fresh fecal samples from at least 1 pig per pen ( $n=7$ pigs per treatment) were collected in sterile tubes, immediately snap-frozen in liquid nitrogen, and stored at $-80^{\circ} \mathrm{C}$ for subsequent analysis of gut microbiota composition and concentrations of organic acids.

\section{Chemical Analysis}

Samples of diets and feces were analyzed for gross energy (GE), dry matter $(\mathrm{DM})$, organic matter $(\mathrm{OM})$, crude protein $(\mathrm{CP})$, ether extract (EE), and total dietary fiber (TDF) according to the AOAC procedure. Gross energy was determined by automatic adiabatic oxygen bomb calorimetry (Parr 1281, Automatic Energy Analyzer, Moline, IL, USA). Acid insoluble ash (AIA) in the diets and feces was measured (De Coca-Sinova et al., 2011). Apparent total tract digestibility of GE, DM, OM, CP, EE, and TDF were determined (Medel et al., 1999).

Insoluble and soluble dietary fiber content of $\mathrm{CB}$ and $\mathrm{FCB}$ were analyzed according to method 991.43 (AOAC International, 2007) using the Ankom TDF Dietary Fiber Analyzer (Ankom Technology, USA). Cellulose, hemicellulose, and lignin content of ingredients were measured (Bertram et al., 2009). Nonstarch polysaccharides and their monosaccharide components were measured on the basis of alditol acetates by gas-liquid chromatography (Aglilent GC 6890, USA) with a column of $30 \mathrm{~m}$ $\times 0.25 \mathrm{~mm} \times 0.25 \mathrm{~mm}$ (Agilent DB-225, USA) at speed of 20 $\mathrm{mL} / \mathrm{min}$. The column temperature was $220^{\circ} \mathrm{C}$ and the injector and detector temperatures were $250^{\circ} \mathrm{C}$.

\section{Extraction of Nucleic Acids and PCR Amplification}

Microbial DNA was extracted from fecal samples using the DNA Kit (Omega Bio-tek, Norcross, GA, USA) according to manufacturer's protocols. The V4-V5 region of the bacteria $16 \mathrm{~S}$ ribosomal RNA gene were amplified by PCR $\left(95^{\circ} \mathrm{C}\right.$ for $2 \mathrm{~min}$, followed by 25 cycles at $95^{\circ} \mathrm{C}$ for $30 \mathrm{~s}, 55^{\circ} \mathrm{C}$ for $30 \mathrm{~s}$, and $72^{\circ} \mathrm{C}$ for $30 \mathrm{~s}$ and a final extension at $72^{\circ} \mathrm{C}$ for $5 \mathrm{~min}$ ) using primers 515F $5^{\prime}$-barcode- GTGCCAGCMGCCGCGG)-3' and 907R 5'-CCGTCAATTCMTTTRAGTTT-3' (Tsai et al., 2011), where barcode is an eight-base sequence unique to each sample. PCR reactions were performed in triplicate in $20 \mu \mathrm{L}$ mixture containing $4 \mu \mathrm{L}$ of $5 \times$ FastPfu Buffer, $2 \mu \mathrm{L}$ of $2.5 \mathrm{mM}$ dNTPs, $0.8 \mu \mathrm{L}$ of each primer $(5 \mu \mathrm{M}), 0.4 \mu \mathrm{L}$ of FastPfu Polymerase, and $10 \mathrm{ng}$ of template DNA.

\section{Illumina Sequencing and Data Analysis}

Amplicons were extracted from 2\% agarose gels and purified using the AxyPrep DNA Gel Extraction Kit (Axygen Biosciences, Union City, CA, USA) and quantified using QuantiFluor ${ }^{\mathrm{TM}}-\mathrm{ST}$ (Promega, USA). Purified amplicons were pooled in equimolar concentrations and paired-end sequenced $(2 \times 300)$ on an Illumina MiSeq platform according to the standard protocols. Raw fastq files were demultiplexed, and quality-filtered using QIIME (version 1.17). Operational taxonomic units (OTUs) were clustered with $97 \%$ similarity cutoff using UPARSE and chimeric sequences were identified and removed using UCHIME. The taxonomy of each 16S rRNA gene sequence was analyzed by RDP Classifier (http://rdp.cme.msu.edu/) against the silva (SSU128) $16 \mathrm{~S}$ rRNA database using confidence threshold of 70\%. Raw data were submitted to NCBI sequence read archive database with the accession No. SRP105389.

\section{Quantification of SCFAs in Fecal Samples}

Fecal sample $(0.5 \mathrm{~g})$ was weighed into a $10 \mathrm{~mL}$ polypropylene tube and $8 \mathrm{~mL}$ deionized water was added. After using an ultrasonic bath for $30 \mathrm{~min}$, the mixture was centrifuged at 8,000 $\times \mathrm{g}$ for $10 \mathrm{~min}$. The suspension was diluted (1:50) with water and filtered through a $0.22 \mu \mathrm{m}$ filter. A $25 \mu \mathrm{L}$ sample solution was extracted and analyzed for the SCFAs, including lactate, acetate, propionate, isobutyrate, isovalerate, and valerate by a high performance ion chromatography (ICS-3000 Dionex, USA) (Hammami et al., 2015). These SCFAs were separated by an AS11 analytical column $(250 \times 4 \mathrm{~mm})$ and an AG11 guard column under the gradient condition: $0-5 \mathrm{~min}, 0.8-1.5 \mathrm{mM} ; 5-10 \mathrm{~min}$, 1.5-2.5 mM, $10-15 \mathrm{~min}, 2.5 \mathrm{mM}$, the flow rate was $1.0 \mathrm{~mL} / \mathrm{min}$. The gradient was carried out with potassium hydroxide.

\section{Statistical Analysis}

Data on growth performance, nutrient digestibility, and fecal organic acid content was performed using SPSS 19.0 (Chicago, IL, USA). The data were analyzed using one-way analysis of variance (ANOVA) followed by Tukey's test, and the results were presented as mean values \pm SEM. Microbiota diversity metrics were performed from normalized OTU reads using $\mathrm{R}$ software (version 3.2.2) (https://www.r-project.org/). The relative abundance of gut microbiota composition (phyla, classes, orders, families, and genera) was analyzed by the Kruskal-Wallis method. Differences were considered significant at $P<0.05$.

\section{RESULTS}

\section{The Chemical Composition of CB and FCB}

Corn bran mainly contained insoluble dietary fiber (Table 1). The contents of cellulose, hemicellulose, and lignin were 23\% lower in FCB than CB; while soluble dietary fiber and nonstarch polysaccharide contents were increased $\sim 53$ and $29 \%$, respectively. The dominant monosaccharide residues of nonstarch polysaccharide in CB and FCB were arabinose, xylose, and glucose. The contents of arabinose, xylose, and glucose in FCB were increased $\sim 50,39$, and $23 \%$, respectively. 
TABLE 1 | Composition of corn bran (CB) and fermented CB (FCB) (g/kg, DM basis) ${ }^{1}$.

\begin{tabular}{lrr}
\hline Items & CB & FCB \\
\hline Starch & 243.2 & 131.3 \\
Organic matter & 971.7 & 978.4 \\
Crude protein & 164.8 & 115.1 \\
Cellulose & 120.1 & 93.1 \\
Hemicellulose & 332.7 & 262.2 \\
Lignin & 22.9 & 11.5 \\
Total dietary fiber & 601.3 & 694.4 \\
SDF & 66.7 & 102.6 \\
IDF & 534.7 & 591.8 \\
SDF/IDF & 12.5 & 17.3 \\
NSP & 535.5 & 689.7 \\
Rhamnose & 3.5 & 2.4 \\
Fructose & 2.0 & 2.9 \\
Ribose & 3.0 & 2.7 \\
Arabinose & 89.5 & 134.2 \\
Xylose & 148.0 & 206.0 \\
Mannose & 18.5 & 15.1 \\
Galactose & 24.5 & 23.3 \\
Glucose & 246.5 & \\
\hline & & \\
\hline SDF solubl. & & \\
\hline
\end{tabular}

${ }^{1} S D F$, soluble dietary fiber; IDF, insoluble dietary fiber; NSP, non-starch polysaccharides.

\section{Growth Performance and Nutrient Digestibility}

There were no differences in ADFI, ADG, and FCR among the dietary treatments (Table 2), which indicated that there are no effect on growth performance when treated by dietary $\mathrm{CB}$ and FCB. The apparent digestibility of GE, DM, CP, EE, and TDF in different groups were detected (Table 3). The apparent digestibility of $\mathrm{OM}$ decreased $(P<0.05)$ in $\mathrm{CB}$ and $\mathrm{FCB}$ treatments compared to CON group. There was no effect of dietary treatment on digestibility of other measured nutrients.

\section{Bacterial Community Richness and Biodiversity}

There were 818,817 , and 787 operational taxonomic units (OTUs) obtained from pigs fed $\mathrm{CON}, \mathrm{CB}$, and FCB dietary treatments, respectively, of which 764 were common OTUs among 3 experimental groups (Figure 1A). Moreover, a total of 24 unique OTUs were found within CON, CB, and FCB groups (11, 11, and 2, respectively). The $\alpha$-diversity index of Chao 1 in the FCB group was lower compared to the CON group $(P<0.05)$, and the Shannon index was not affected by dietary treatments (Figures 1B,C).

Fifteen distinct phylum were identified in feces of finishing pigs fed different CB supplementations (Supplemental Table 2), where Firmicutes and Bacteroidetes accounted for $\sim 94 \%$ within all dietary treatments (Figure 2A). The study detected Fibrobacteres phylum, which is associated with cellulolytic bacteria, and there was no difference between treatments where relative abundance in $\mathrm{CON}, \mathrm{CB}$, and $\mathrm{FCB}$ groups was $0.04,0.13$, and $0.09 \%$, respectively (Supplemental
TABLE 2 | Effects of dietary corn bran (CB) and fermented CB (FCB) on growth performance of finishing pigs ${ }^{1}$.

\begin{tabular}{lcccc}
\hline Items & \multicolumn{4}{c}{ Dietary treatments } \\
\cline { 2 - 5 } & CON & CB & FCB & $P$-value \\
\hline ADFl, kg & $2.44 \pm 0.19$ & $2.60 \pm 0.13$ & $2.51 \pm 0.09$ & 0.751 \\
ADG, g & $852 \pm 64.7$ & $897 \pm 25.6$ & $856 \pm 27.0$ & 0.726 \\
FCR & $2.88 \pm 0.15$ & $2.92 \pm 0.14$ & $2.99 \pm 0.10$ & 0.844
\end{tabular}

${ }^{1}$ Twenty pigs per treatment (5 pens of 4 pigs) were measured for growth performance in a $21 d$ feeding trial. The results were presented as mean values \pm SEMs. Data were analyzed by one-way ANOVA with Tukey's test. CON, control group; ADFI, average daily feed intake; ADG, average daily gain; FCR, feed conversion ratio.

TABLE 3 | Effect of dietary corn bran (CB) and fermented CB (FCB) on nutrient digestibility of finishing pigs ${ }^{1}$.

\begin{tabular}{|c|c|c|c|c|}
\hline \multirow[t]{2}{*}{ Items, \% } & \multicolumn{3}{|c|}{ Dietary treatments } & \multirow[b]{2}{*}{$P$-value } \\
\hline & CON & CB & FCB & \\
\hline GE & $88.58 \pm 0.53$ & $87.43 \pm 0.42$ & $87.68 \pm 0.16$ & 0.194 \\
\hline DM & $89.46 \pm 0.49$ & $88.27 \pm 0.37$ & $88.50 \pm 0.12$ & 0.123 \\
\hline OM & $90.91 \pm 0.44^{a}$ & $89.40 \pm 0.40^{b}$ & $89.70 \pm 0.12^{b}$ & 0.039 \\
\hline $\mathrm{CP}$ & $86.73 \pm 0.64$ & $86.40 \pm 0.51$ & $87.85 \pm 0.28$ & 0.085 \\
\hline EE & $64.05 \pm 1.51$ & $67.17 \pm 1.32$ & $65.94 \pm 1.22$ & 0.188 \\
\hline TDF & $62.12 \pm 1.43$ & $63.87 \pm 0.94$ & $64.75 \pm 1.38$ & 0.203 \\
\hline
\end{tabular}

${ }^{1} \mathrm{Fecal}$ samples from 1 pig per pen ( $n=5$ pigs per treatment) performed for nutrient digestibility in a $21 d$ feeding trial. The results were presented as mean values \pm SEMs. Data were analyzed by one-way ANOVA with Tukey's test. Different superscript lowercase letters within each row mean significantly different $(P<0.05)$. CON, control group; GE, gross energy; $D M$, dry matter; $O M$, organic matter; $C P$, crude protein; $E E$, ether extract; $T D F$, total dietary fiber.

Table 2). The dominant families within the Firmicutes phylum consisted of Clostridiaceae_1, Veillonellaceae, Ruminococcaceae, Lachnospiraceae, Streptococcaceae, and Peptostreptococcaceae, while the main families within the Bacteridetes phylum were Prevotellaceae, Bacteroidales_S24-7_group, and Rikenellaceae (Figure 2B). The relative abundance of bacterial communities at the family level did not differ among experimental groups (Supplemental Table 3). The relative abundance of the top 30 genera bacteria community were analyzed (Figure 2C), and their relative abundance were higher than $1 \%$ at least 1 treatment group (Supplemental Table 4). The population of Lachnospiraceae_NK4A136_group in CB treatment $(1.29 \%)$ was higher than CON $(0.92 \%)$ and FCB $(0.26 \%)$ groups $(P<0.05)$. The relative abundance of norank $\_\_$Prevotellaceae was higher in FCB group (1.47\%) compared to CON $(0.69 \%)$ and CB $(0.64 \%)$ groups $(P<0.05)$. In addition, pigs fed CON diet $(2.24 \%)$ had higher abundance of Rikenellaceae_RC9_gut_group compared to $\mathrm{CB}(1.61 \%)$ and $\mathrm{FCB}(1.17 \%)$ treatments $(P<0.05)$.

\section{SCFAs in Fecal Samples}

Lactic acid was not detected in the majority of fecal samples, and the SCFAs including acetate, priopinate, isobutyrate, butyrate, isovalerate, and valerate were not affected by the $\mathrm{CB}$ or $\mathrm{FCB}$ dietary treatments (Table 4), which suggested that SCFAs, the 
A
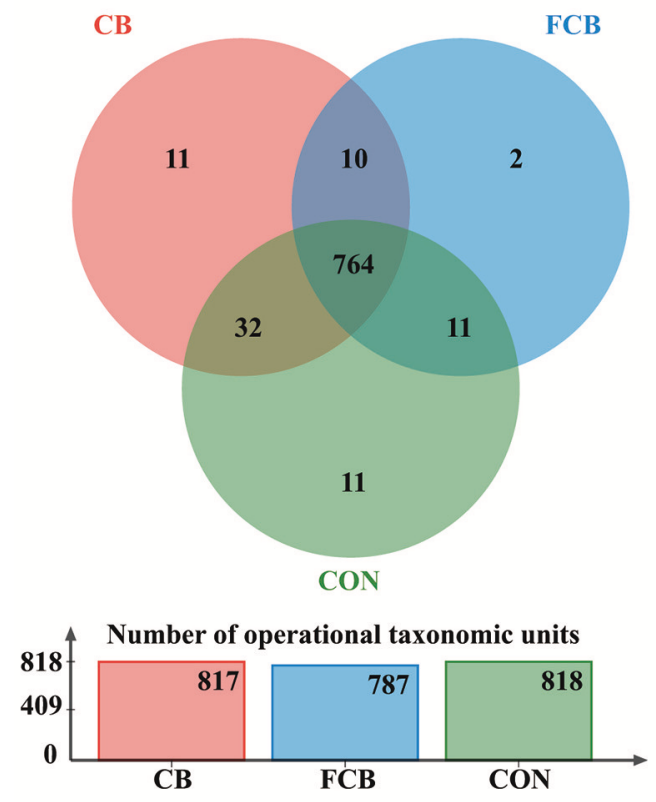

B

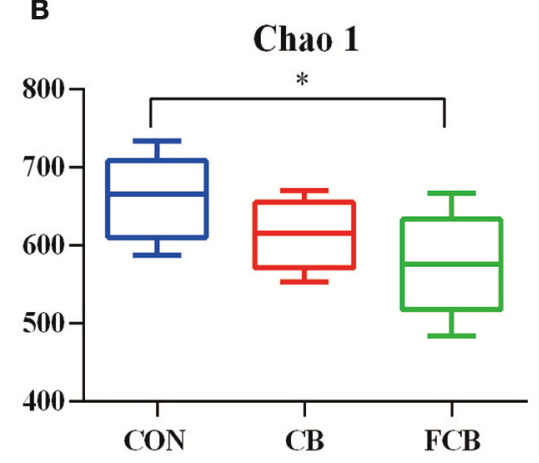

C Shannon

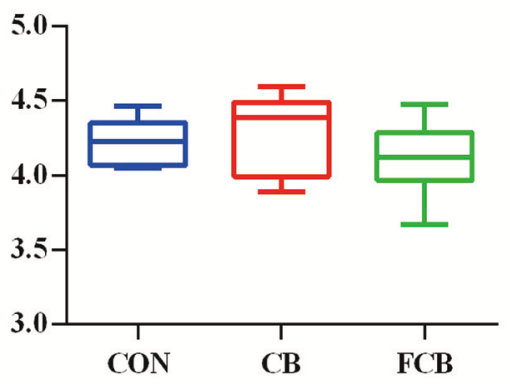

FIGURE 1 | Effect of dietary corn bran (CB) and fermented CB (FCB) on intestinal microbiota richness in finishing pigs. (A) OTU Venn of 3 dietary treatments. (B) The Chao 1 index of bacterial community. (C) Shannon index of bacterial community. Fecal samples from 7 pigs per treatment were performed for $16 S$ rRNA gene amplicon sequencing analysis in a $21 \mathrm{~d}$ feeding trial. The results were analyzed by Kruskal-Wallis $\mathrm{H}$ test and presented as mean values of different bacteria, and asterisk means $P<0.05$. CON, control group.

microbial metabolites, may not mediate the effects of dietary CB or FCB.

\section{DISCUSSION}

This study was performed to compare the effect of $\mathrm{CB}$ and FCB inclusion on growth performance, nutrient digestibility, and gut microbiota composition in finishing pigs. Dietary fiber is involved in various physiological processes and plays an essential role in enhancing immune regulation and gut health (Koh et al., 2016). Corn bran is the main by-products from the corn milling process, and is commonly utilized as a feed ingredient for animals. The majority of plant polysaccharides like $\mathrm{CB}$ cannot be digested directly by enzymes in humans and monogastric animals, but they can be degraded by colonic bacterial communities (Sonnenburg and Sonnenburg, 2014).

Polysaccharide utilization and fiber digestibility can be improved with special processing technology. Solid-state fermentation of feedstuffs has been applied for the purpose of modifying biological materials to reduce the insoluble fiber content of feeds resulting in useful feedstuffs (Pallin et al., 2016), because the fibrolytic enzymes produced from bacteria, such as cellulases and hemicellulases, are able to degrade complex polysaccharides (Kracher et al., 2016). In this study, the contents of cellulose and hemicellulose were reduced in the FCB. Meanwhile, the level of soluble dietary fiber increased after in vitro fermentation compared with $\mathrm{CB}$. Chemical analysis demonstrated that the solid-state fermentation makes the insoluble glycans in corn bran easily digestible. In addition, 3 monosaccharide residues (arabinose, xylose, and glucose) were increased, and these should be closely associated with the reaction of fermentation by the mixed cultures of Bacillus subtilis MA139, E. faecium, and S. cerevisae. It has been reported that arabinose and xylose are major sugars in the plant cell wall of wheat bran and CB (Rose et al., 2010; Chen H. et al., 2015); B. subtilis produce xylanases, which are able to degrade soluble and insoluble xylan substrates to acidic xylo-oligosaccharides (Rhee et al., 2014). The strain of B. subtilis MA139 used in this study was identified and has been used as a potential probiotic to improve pig performance (Yang et al., 2015). Further, we found an innovative strategy to apply B. subtilis MA139 with lactic acid bacteria to produce solid-state fermentation of feedstuffs. These co-inoculating strains showed high antimicrobial activity against on Enterobacteriaceae, such as E. coli and S. typhimurium. Lactic acid bacteria, such as Lactobacillus reuteri and Lactobacillus fermentium, have been applied for feed fermentation due to their beneficial effects on preservation of feeds, favorable aroma, and flavor of bacterial end-products (Pallin et al., 2016). In the present study, E. faecium was applied in solid-state fermentation in $\mathrm{CB}$ because it can synthesize enzymes to degrade starch and the 

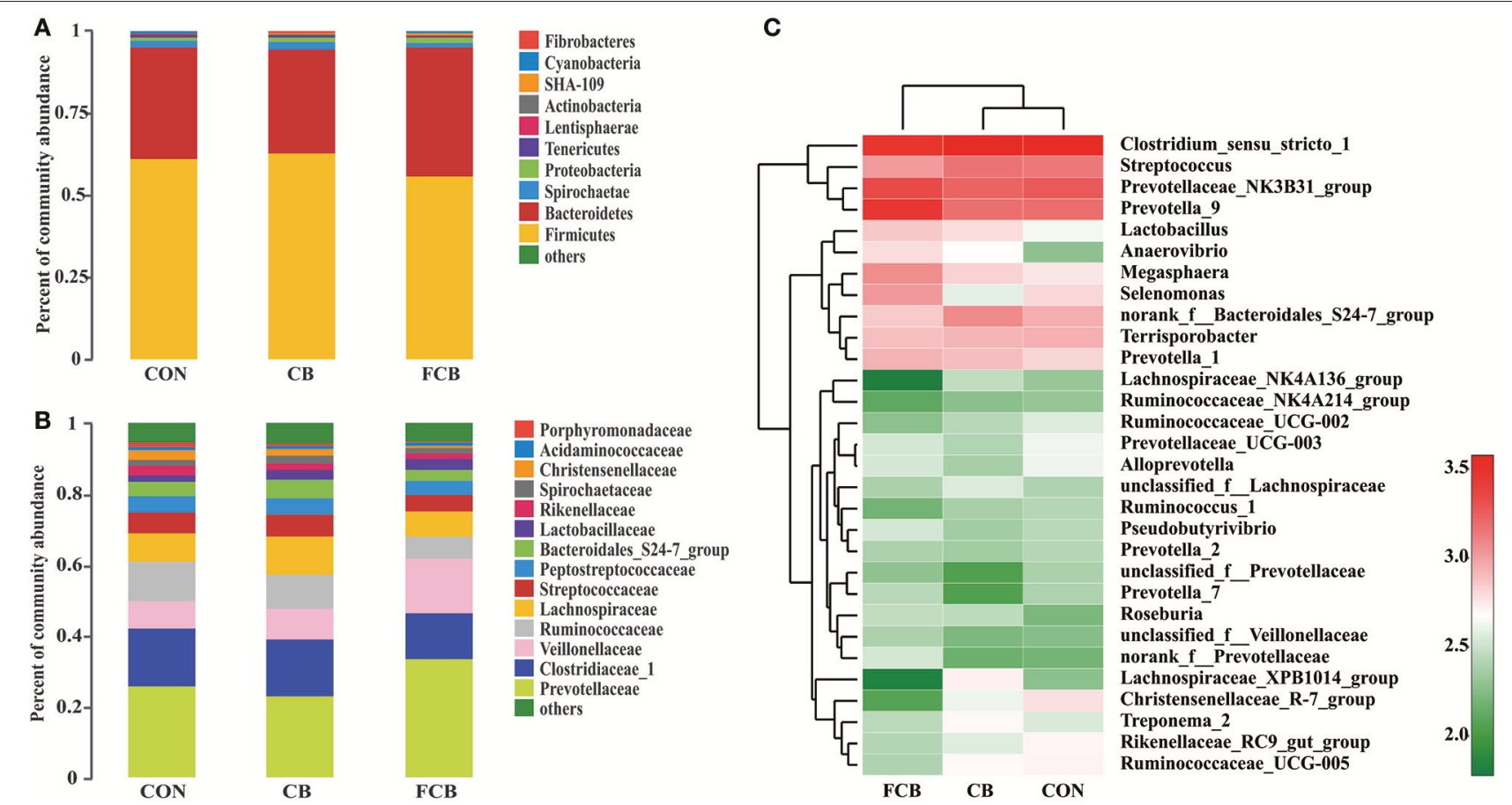

FIGURE 2 I Effect of dietary corn bran (CB) and fermented CB (FCB) on gut microbiota composition in finishing pigs. (A) Microbial community bar plot at the phylum level with the relative abundance higher than $0.05 \%$. (B) Microbial community bar plot at the family level with the relative abundance higher than $1 \%$. (C) Microbial community heat map of the top 30 bacteria at the genus level. Fecal samples from 7 pigs per treatment were performed for 165 rRNA gene amplicon sequencing analysis in a $21 \mathrm{~d}$ feeding trial. The results were analyzed by Kruskal-Wallis $\mathrm{H}$ test and presented as mean percentage of different bacteria. CON, control group.

bacterial metabolite of lactic acid is beneficial for modulating immune response of the host (Siepert et al., 2014; Starke et al., 2015).

Dietary fiber plays an important role in maintaining bacterial diversity and intestinal homeostasis (Chen J. et al., 2015). It is reported that high concentrations of dietary fiber result in body weight loss (Kovatcheva-Datchary et al., 2015), which may be attributed to an improvement in satiety due to fiber viscosity. Fiber-rich diets may affect satiety through an increase in mastication or changes in gut hormones levels, such as glucagonlike peptide 1 (Näslund and Hellström, 2007). A previous study showed $\mathrm{CB}$ resulted in a lesser desire for food intake in humans (Rose et al., 2010). In the present study, the pigs fed CB or FCB treatments did not show significant differences in daily feed intake and average daily gain and digestibility of organic matter decreased in both $\mathrm{CB}$ and FCB treatments compared to CON group. This observation was in agreement with the previous studies in pigs (Bindelle et al., 2008; Bertram et al., 2009). The result suggests that diets containing $10 \% \mathrm{CB}$ or FCB did not affect satiety response in finishing pigs over $21 \mathrm{~d}$ study.

The gut bacterial community and the abundance of bacterial metabolites are influenced by diets, in turn, the gut microbiota composition impacts nutrient digestibility (Fan et al., 2015, 2017a). Dietary fiber is characterized by different monosaccharides within complex polymers of $\beta$-1,4-linked units of hexose (cellobiose and glucose) and/or pentose (arabinose and xylose) sugars (Martin et al., 1998), which cannot be digested in the small intestine of monogastric animals due to a lack of cellulolytic enzymes. However, the most dense and diverse bacterial community inhabits the large intestine and can produce a range of cellulolytic enzymes to degrade different fiber component in diet. Consequently, the activity of bacterial physiology relates to the fiber metabolism in the gut. The degradation of complex carbohydrates requires a range of bacterial enzymatic activities, normally accomplished by a consortium of bacteria rather than a single species (Scott et al., 2008).

There is a considerable degree in variation of the intestinal microbiota composition between studies, but Firmicutes and Bacteroidetes were the most dominant phyla in healthy individuals (Flint et al., 2007). In our study, the Firmicutes and Bacteriodetes were numerically dominant in finishing pigs with the abundance higher than 94\%, and the result is consistent with fecal microbiota from pigs reported in other studies observed (Heinritz et al., 2016). It has been reported that fiber-rich diets result in increased abundance of Bacteroidetes, especially the genera of Prevotella and Xylanbacter (De Filippo et al., 2010; Trompette et al., 2014). For instance, $B$. thetaiotaomicron is regarded as a glycan-degrading generalist, which can degrade complex glycan of plant wall, animal tissue, and host mucin (Koropatkin et al., 2012). The change in the ratio of Firmicutes and Bacteroidetes in FCB group might be due to the glycan composition in FCB. Addition to Firmicutes and Bacteroidetes phylum, there were 13 other phylum detected 
TABLE 4 | Effect of dietary corn bran (CB) and fermented CB (FCB) on short-chain fatty acids concentrations in feces of finishing pigs ${ }^{1}$.

\begin{tabular}{lcccc}
\hline Item, $\mathbf{m g} / \mathbf{g}$ & \multicolumn{4}{c}{ Dietary treatments } \\
\cline { 2 - 5 } & CON & CB & FCB & P-value \\
\hline Acetate & $4.07 \pm 0.17$ & $4.26 \pm 0.18$ & $3.90 \pm 0.10$ & 0.289 \\
Propionate & $2.43 \pm 0.17$ & $2.60 \pm 0.16$ & $2.50 \pm 0.09$ & 0.682 \\
Isobutyrate & $0.35 \pm 0.06$ & $0.26 \pm 0.02$ & $0.25 \pm 0.02$ & 0.185 \\
Butyrate & $2.00 \pm 0.22$ & $1.68 \pm 0.16$ & $1.62 \pm 0.09$ & 0.259 \\
Isovalerate & $0.23 \pm 0.04$ & $0.20 \pm 0.01$ & $0.19 \pm 0.01$ & 0.352 \\
Valerate & $0.52 \pm 0.08$ & $0.41 \pm 0.04$ & $0.46 \pm 0.04$ & 0.388 \\
Total SCFAs & $9.05 \pm 0.52$ & $9.51 \pm 0.48$ & $8.84 \pm 0.16$ & 0.493 \\
\hline
\end{tabular}

${ }^{1}$ Fecal samples from at least 1 pig per pen ( $n=7$ pigs per treatment) were performed for SCFAs analysis in a $21 d$ feeding trial, data were presented as mean values \pm SEMs. Data were analyzed by one-way ANOVA with Tukey's test. CON, control group; SCFAs, short-chain fatty acids.

among the 3 dietary treatments, including Spirochaetae, Proteobacteria, Tenericutes, Lentisphaerae, Actinobacteria, SHA-109, Cyanobacteria, Fibrobacteres, Saccharibacteria, unclassified_k_norank, Synergistetes, Elusimicrobia, and Chlamydiae. Compared to a previous study on gut microbiota composition in different age of pigs (Niu et al., 2015), wherein the SHA-109, Saccharibacteria, unclassified_k_norank, and Elusimicrobia were unique phylum, and this might be associated with different experiment diets between the studies. Specifically, Fibrobacteres phylum was identified by gene sequencing, albeit in a small proportion. The genus Fibrobacter belongs to the poorly defined Fibrobacteres phylum, which was regarded as Spirochetes or Bacteroidetes. In fact, the Fibrobacteres phylum is characterized by high cellulolytic activity and capable of degrading refractory plant structural polysaccharides and they play a prominent function in fiber-digesting and energy metabolism in gastrointestinal tract (Qi et al., 2005). In the rumen, the genus Fibrobacter is responsible for the degradation of dietary fiber and polysaccharides. The increased abundance of Fibrobacter resulted in more sugars and SCFAs in gastrointestinal tract of dairy calves (Deng et al., 2017). Moreover, Fibrobacteres was observed in different ages of pig; however, diet composition and fiber components were not mentioned (Niu et al., 2015). The mechanistic link between Fibrobacteres and polysaccharide metabolism needs further study.

Herein, the relative abundance of the top 30 bacterial communities at the genus level was shown in a heat map. Clostridium has been associated with dietary fiber metabolism previously (Niu et al., 2015). Lachnospiraceae and Rumininococcaceae are two dominant bacteria from Clostridia among the 3 dietary treatments and are regarded as fibrolytic specialists of commensal bacteria to degrade the complex plant material involved in activating carbohydrate enzymes, sugar transport, and metabolic pathways. Therefore, Lachnospiraceae and Rumininococcaceae are associated with the maintenance of gut health (Biddle et al., 2013). In this study, the CB group showed the greatest abundance of genus Lachnospiraceae_NK4A136_group and Lachnospiraceae_XPB1014_group. This was attributed to the dietary inclusion of $\mathrm{CB}$ which can stimulate fibrolytic bacteria to degrade the complex plant bran of recalcitrant substrate (Brulc et al., 2009). In contrast, non-adherent Bacteriodes sp. and Bifidobacterium sp. ferment easily hydrolysable starch (Cameron et al., 2014). Pigs fed CB treatment showed distinctly more bacteria in the Lachnospiraceae and Rumininococcaceae families of the Firmicutes phylum, while Prevotellaceae of the Bacteroidetes phylum was more abundant in the FCB group. The relative abundance of Lactobacillus and Anaerovibrio were higher in both CB and FCB groups compared to CON group. These genera are able to utilize glycerol and have been associated with propionate and butyrate production (Minuti et al., 2015). The result was supported by a previous study where insoluble dietary fiber extracted from $\mathrm{CB}$ stimulated the growth of beneficial bacteria in the gut of health individuals (Hsu et al., 2004). The Prevotellaceae family within Bacteroidetes phylum are involved in glucose metabolism (Heinritz et al., 2016) and the relative abundance of norank_f_Prevotellaceae was significantly higher in FCB group compared to the other 2 groups, consistent with the increased levels of soluble dietary fiber and glucose in FCB. Rikenellaceae are commensal bacteria in the gut in relatively small proportion, which can thrive on high-fat diets and are enriched in gut microbiota of the obese. A study reported that the abundance of Rikenellaceae_RC9_gut_group increased with high concentration of dietary protein in pigs (Fan et al., 2017a,b). In our study, the proportion of Rikenellaceae_RC9_gut_group decreased in $\mathrm{CB}$ and FCB treatments compared to the CON group, which suggested that Rikenellaceae_RC9_gut_group have an impact on carbohydrate and lipid metabolism. The alterations in microbial populations with the inclusion of $\mathrm{CB}$ and $\mathrm{FCB}$ are consistent with the relative fermentable substrate composition of feedstuff.

The end-product of bacterial metabolism plays an important role in shaping the bacterial community. Bacterial metabolite production is dependent on the available substrates, but in general, most end-products detectable in feces are acetate, propionate, and butyrate (Huang et al., 2015). Fermentation of different carbohydrate substrates by bacterial consortia normally results in variations of SCFAs production. Many colonic bacteria produce lactate as a fermentation product, but the concentration of lactate was $<3-5 \mathrm{mM}$ in fecal sample (Duncan et al., 2007). Lactic acid was not detected in the majority of fecal samples in our study. Lactate-utilizing bacteria includes Eubacterium hallii and Anaerostipes caccae, which can be detected in fecal samples from healthy individuals (Duncan et al., 2004). The species of $E$. hallii and $A$. caccae were identified by $16 \mathrm{~S}$ rRNA sequencing in the present study, but their abundance were low and not affected by dietary treatments. Butyrate as one of the important bacterial metabolites that can provide energy for colonic epithelium, and also plays key functions in protection against colonic diseases (Koh et al., 2016). Eubacterium rectale and Roseburia species are two important groups of butyrate-producing bacteria. In the present study, the abundance of Roseburia was not influenced by dietary treatments, and a population of E. rectale was not observed in fecal samples. There were few changes on gut bacteria related to SCFAs production, and this observation was consistent with the stable SCFA concentration among the treatment groups. 
In conclusion, diets containing $\mathrm{CB}$ and $\mathrm{FCB}$ did not impact growth performance or nutrient digestibility in finishing pigs. Gut microbiota composition was affected slightly by dietary treatments, as the bacterial richness and diversity in FCB was decreased compared to control and $\mathrm{CB}$ groups. Moreover, the relative abundance of bacteria related to cellulolytic degradation was higher in $\mathrm{CB}$ fed pigs; whereas FCB fed pigs had greater proportions of starch-fermenting bacteria. The differences in bacterial community between $\mathrm{CB}$ and FCB inclusion might be due to the alteration on chemical component of polysaccharides via in vitro fermentation. Thus, the underlying mechanisms on how the chemical characterizations of corn bran components influence the bacteria related to polysaccharide and lipid metabolism still needs further investigation.

\section{AUTHOR CONTRIBUTIONS}

XM, and WL: Designed the experiments; PL, JinZ, PG, and ZG: Performed the experiments; LL and CW: Analyzed the data; PL: Wrote the manuscript, which was edited by CL, LJ, SQ, JieZ, and XM. XM: Resourced the project. All authors read and approved the final manuscript.

\section{REFERENCES}

AOAC International (2007). Official Methods of Analysis of AOAC International. Gaithersburg, MD: AOAC International.

Bertram, H. C., Malmendal, A., Nielsen, N. C., Straadt, I. K., Larsen, T., Knudsen, K. E., et al. (2009). NMR-based metabonomics reveals that plasma betaine increases upon intake of high-fiber rye buns in hypercholesterolemic pigs. Mol. Nutr. Food Res. 53, 1055-1062. doi: 10.1002/mnfr.200800344

Biddle, A., Stewart, L., Blanchard, J., and Leschine, S. (2013). Untangling the genetic basis of fibrolytic specialization by Lachnospiraceae and Ruminococcaceae in diverse gut communities. Diversity 5, 627-640. doi: $10.3390 / \mathrm{d} 5030627$

Bindelle, J., Leterme, P., and Buldgen, A. (2008). Nutritional and environmental consequences of dietary fibre in pig nutrition: a review. Biotechnol. Agron. Soc. Environ. 12, 69-80. Available online at: http://popups.ulg.ac.be/1780-4507/ index.php?id $=2179$

Brulc, J. M., Antonopoulos, D. A., Miller, M. E., Wilson, M. K., Yannarell, A. C., Dinsdale, E. A., et al. (2009). Gene-centric metagenomics of the fiber-adherent bovine rumen microbiome reveals forage specific glycoside hydrolases. Proc. Natl. Acad. Sci. U.S.A. 106, 1948-1953. doi: 10.1073/pnas.0806191105

Cameron, E. A., Maynard, M. A., Smith, C. J., Smith, T. J., Koropatkin, N. M., and Martens, E. C. (2014). Multidomain carbohydrate-binding proteins involved in Bacteroides thetaiotaomicron starch metabolism. J. Biol. Chem. 287, 34614-34625. doi: 10.1074/jbc.M112.397380

Chen, H., Wang, W., Degroote, J., Possemiers, S., Chen, D. W., De Smet, S., et al. (2015). Arabinoxylan in wheat is more responsible than cellulose for promoting intestinal barrier function in weaned male piglets. J. Nutr. 145, 51-58. doi: $10.3945 /$ jn.114.201772

Chen, J., Li, Y., Tian, Y., Huang, C., Li, D., Zhong, Q., et al. (2015). Interaction between microbes and host intestinal health: modulation by dietary nutrients and gut-brain-endocrine-immune axis. Curr. Protein Pept. Sci. 16, 592-603. doi: 10.2174/1389203716666150630135720

De Coca-Sinova, A., Mateos, G. G., Gonzalez-Alvarado, J. M., Centeno, C., Lazaro, R., and Jimenez-Moreno, E. (2011). Comparative study of two analytical procedures for the determination of acid insoluble ash for evaluation of nutrient retention in broilers. Spanish J. Agric. Res. 9, 761-768. doi: $10.5424 /$ sjar/20110903-439-10

De Filippo, C., Cavalieri, D., Di Paola, M., Ramazzotti, M., Poullet, J. B., Massart, S., et al. (2010). Impact of diet in shaping gut microbiota revealed by a comparative

\section{FUNDING}

This work was supported by the National Key R\&D Program of China (2017YFD0500501), College of Animal Science and Technology "Young Talents Cultivation Program" in China Agricultural University (2017DKA001), the National Natural Science Foundation of China (31722054, 31472101, and 31528018), the 111 Project (B16044), the National Department Public Benefit Research Foundation (201403047) and the developmental fund for animal science by Shenzhen Jinxinnong Feed Co., Ltd.

\section{ACKNOWLEDGMENTS}

We thank Dr. Xiangli Sun, Zhixiang Sun and Yao Li in Ministry of Agriculture Feed Industry Centre, for the excellent assistance in chemical analysis.

\section{SUPPLEMENTARY MATERIAL}

The Supplementary Material for this article can be found online at: https://www.frontiersin.org/articles/10.3389/fcimb. 2017.00526/full\#supplementary-material

study in children from Europe and rural Africa. Proc. Natl. Acad. Sci. U.S.A. 107, 14691-14696. doi: 10.1073/pnas.1005963107

Deng, Y. F., Wang, Y. J., Zou, Y., Azarfar, A., Wei, X. L., Ji, S. K., et al. (2017). Influence of dairy by-product waste milk on the microbiomes of different gastrointestinal tract components in pre-weaned dairy calves. Sci. Rep. 7:42689. doi: $10.1038 /$ srep 42689

Desai, M. S., Seekatz, A. M., Koropatkin, N. M., Kamada, N., Hickey, C. A., Wolter, M., et al. (2016). A dietary fiber-deprived gut microbiota degrades the colonic mucus barrier and enhances pathogen susceptibility. Cell 167, 1339-1353. doi: 10.1016/j.cell.2016.10.043

Duncan, S. H., Belenguer, A., Holtrop, G., Johnstone, A. M., Flint, H. J., and Lobley, G. E. (2007). Reduced dietary intake of carbohydrates by obese subjects results in decreased concentrations of butyrate and butyrate-producing bacteria in feces. Appl. Environ. Microb. 73, 1073-1078. doi: 10.1128/AEM.02340-06

Duncan, S. H., Louis, P., and Flint, H. J. (2004). Lactate-utilizing bacteria, isolated from human feces, that produce butyrate as a major fermentation product. Appl. Environ. Microb. 70, 5810-5817. doi: 10.1128/AEM.70.10.5810-5817.2004

Fan, P., Li, L., Rezaei, A., Eslamfam, S., Che, D., and Ma, X. (2015). Metabolites of dietary protein and peptides by intestinal microbes and their impacts on gut. Curr. Protein Pept. Sci. 16, 646-654. doi: 10.2174/1389203716666150630133657

Fan, P., Liu, P., Song, P., Chen, X., and Ma, X. (2017a). Moderate dietary protein restriction alters the composition of gut microbiota and improves ileal barrier function in adult pig model. Sci. Rep. 7:43412. doi: 10.1038/srep43412

Fan, P., Song, P., Li, L., Huang, C., Chen, J., Ma, X., et al. (2017b). Roles of biogenic amines in intestinal signaling. Curr. Protein Pept. Sci. 18, 532-540. doi: 10.2174/1389203717666160627073048

Flint, H. J., Duncan, S. H., Scott, K. P., and Louis, P. (2007). Interactions and competition within the microbial community of the human colon: links between diet and health. Environ. Microbiol. 9, 1101. doi: 10.1111/j.1462-2920.2007.01281.x

Gerritsen, R., van Der Aar, P., and Molist, F. (2012). Insoluble nonstarch polysaccharides in diets for weaned piglets. J. Anim. Sci. 90(Suppl. 4), 318-320. doi: $10.2527 /$ jas. 53770

Hammami, R., Bédard, F., Gomaa, A., Subirade, M., Biron, E., and Fliss, I. (2015). Lasso-inspired peptides with distinct antibacterial mechanisms. Amino Acids 47, 417-428. doi: 10.1007/s00726-014-1877-x

Han, M., Wang, C., Liu, P., Li, D., Li, Y., and Ma, X. (2017). Dietary fiber gap and host gut microbiota. Protein Pept. Lett. 24, 388-396. doi: 10.2174/0929866524666170220113312 
Hashemi, Z., Fouhse, J., Im, H., Chan, C., and Willing, B. (2017). Dietary pea fiber supplementation improves glycemia and induces changes in the composition of gut microbiota, serum short chain fatty acid profile and expression of mucins in glucose intolerant rats. Nutrients 12, 11. doi: 10.3390/nu9111236

Hedemann, M. S., Theil, P. K., and Bach Knudsen, K. E. (2009). The thickness of the intestinal mucous layer in the colon of rats fed various sources of nondigestible carbohydrates is positively correlated with the pool of SCFA but negatively correlated with the proportion of butyric acid in digesta. Br. J. Nutr. 102, 117-125. doi: 10.1017/S0007114508143549

Heinritz, S. N., Weiss, E., Eklund, M., Aumiller, T., Louis, S., Rings, A., et al. (2016). Intestinal microbiota and microbial metabolites are changed in a pig model fed a high-fat/low-fiber or a low-fat/high-fiber diet. PLOS ONE 11:e0154329. doi: 10.1371/journal.pone.0154329

Horita, M., Kitamoto, H., Kawaide, T., Tachibana, Y., and Shinozaki, Y. (2015). Onfarm solid state simultaneous saccharification and fermentation of whole crop forage rice in wrapped round bale for ethanol production. Biotechnol. Biofuels 30, 9. doi: 10.1186/s13068-014-0192-9

Hsu, C. K., Liao, J. W., Chung, Y. C., Hsieh, C. P., and Chan, Y. C. (2004). Xylooligosaccharides and fructooligosaccharides affect the intestinal microbiota and precancerous colonic lesion development in rats. J. Nutr. 134, $1523-1528$.

Huang, C., Song, P., Fan, P., Hou, C., Thacker, P., and Ma, X. (2015). Dietary sodium butyrate decreases postweaning diarrhea by modulating intestinal permeability and changing the bacterial communities in weaned piglets. J. Nutr. 145, 2774-2780. doi: 10.3945/jn.115.217406

Jaworski, N. W., Owusu-Asiedu, A., Walsh, M. C., Mccann, J. C., Loor, J. J., and Stein, H. H. (2017). Effects of a 3 strain -based direct-fed microbial and dietary fiber concentration on growth performance and expression of genes related to absorption and metabolism of volatile fatty acids in weanling pigs. J. Anim. Sci. 95, 308-319. doi: $10.2527 /$ jas2016.0557

Koh, A., De Vadder, F., Kovatcheva-Datchary, P., and Bäckhed, F. (2016). From dietary fiber to host physiology: short-chain fatty acids as key bacterial metabolites. Cell 165, 1332-1345. doi: 10.1016/j.cell.2016.05.041

Koropatkin, N. M., Cameron, E. A., and Martens, E. C. (2012). How glycan metabolism shapes the human gut microbiota. Nat. Rev. Microbiol. 10, 323-335. doi: $10.1038 /$ nrmicro2746

Kovatcheva-Datchary, P., Nilsson, A., Akrami, R., Lee, Y. S., De Vadder, F., Arora, T., et al. (2015). Dietary fiber-induced improvement in glucose metabolism is associated with increased abundance of Prevotella. Cell Metab. 22, 971-982. doi: 10.1016/j.cmet.2015.10.001

Kracher, D., Scheiblbrandner, S., Felice, A. K., Breslmayr, E., Preims, M., Ludwicka, K., et al. (2016). Extracellular electron transfer systems fuel cellulose oxidative degradation. Science 352, 1098-1101. doi: 10.1126/science.aaf3165

Ma, N., Tian, Y., Wu, Y., and Ma, X. (2017). Contributions of the interaction between dietary protein and gut microbiota to intestinal health. Curr. Protein Pept. Sci. 18, 795-808. doi: 10.2174/1389203718666170216153505

Martens, E. C. (2016). Fibre for the future. Nature 529, 158-159. doi: $10.1038 / 529158 \mathrm{a}$

Martin, S. A., Morrison, W. H., and Akin, D. E. (1998). Fermentation of maize bran, oat bran, and wheat bran by Bacteroides ovatus V975. Curr. Microbiol. 36, 90-95. doi: 10.1007/s002849900285

Medel, P., Salado, S., Jcde, B., and Mateos, G. G. (1999). Processed cereals in diets for early-weaned piglets. Anim. Feed Sci. Technol. 82, 145-156. doi: 10.1016/S0377-8401(99)00111-X

Minuti, A., Palladino, A., Khan, M. J., Alqarni, S., Agrawal, A., PiccioliCapelli, F., et al. (2015). Abundance of ruminal bacteria, epithelial gene expression, and systemic biomarkers of metabolism and inflammation are altered during the peripartal period in dairy cows. J. Dairy Sci. 98, 8940-8951. doi: 10.3168/jds.2015-9722

Morowitz, M., Di Caro, V., Pang, D., Cummings, J., Firek, B., Rogers, M., et al. (2017). Dietary supplementation with nonfermentable fiber alters the gut microbiota and confers protection in murine models of sepsis. Crit. Care Med. 45, e516-e523. doi: 10.1097/CCM.0000000000002291

Näslund, E., and Hellström, P. M. (2007). Appetite signaling: from gut peptides and enteric nerves to brain. Physiol. Behav. 92, 256-262. doi: 10.1016/j.physbeh.2007.05.017
Niu, Q., Li, P., Hao, S., Zhang, Y., Kim, S. W., Li, H., et al. (2015). Dynamic distribution of the gut microbiota and the relationship with apparent crude fiber digestibility and growth stages in pigs. Sci. Rep. 5:9938. doi: $10.1038 /$ srep09938

Pallin, A., Agback, P., Jonsson, H., and Roos, S. (2016). Evaluation of growth, metabolism and production of potentially bioactive components during fermentation of barley with Lactobacillus reuteri. Food Microbiol. 57, 159-171. doi: $10.1016 /$ j.fm.2016.02.011

Qi, M., Nelson, K. E., Daugherty, S. C., Nelson, W. C., Hance, I. R., Morrison, M., et al. (2005). Novel molecular features of the fibrolytic intestinal bacterium fibrobacter intestinalis not shared with fibrobacter succinogenes as determined by suppressive subtractive hybridization. J. Bacteriol. 187, 3739-3751. doi: 10.1128/JB.187.11.3739-3751.2005

Rhee, M. S., Wei, L., Sawhney, N., Rice, J. D., John, F. J. S., Hurlbert, J. C., et al. (2014). Engineering the xylan utilization system in Bacillus subtilis for production of acidic xylooligosaccharides. Appl. Environ. Microbiol. 80, 917-927. doi: 10.1128/AEM.03246-13

Rose, D. J., Inglett, G. E., and Liu, S. X. (2010). Utilisation of corn (Zea mays) bran and corn fiber in the production of food components. J. Sci. Food Agric. 90, 915-924. doi: 10.1002/jsfa.3915

Scott, K. P., Duncan, S. H., and Flint, H. J. (2008). Dietary fibre and the gut microbiota. Nutr. Bull. 33, 201-211. doi: 10.1111/j.1467-3010.2008.00706.x

Siepert, B., Reinhardt, N., Kreuzer, S., Bondzio, A., Twardziok, S., Brockmann, G., et al. (2014). Enterococcus faecium NCIMB 10415 supplementation affects intestinal immune-associated gene expression in post-weaning piglets. Vet. Immunol. Immunopathol. 157, 65-77. doi: 10.1016/j.vetimm.2013.10.013

Sonnenburg, E. D., and Sonnenburg, J. L. (2014). Starving our microbial self: the deleterious consequences of a diet deficient in microbiota-accessible carbohydrates. Cell Metab. 20, 779-786. doi: 10.1016/j.cmet.2014.07.003

Starke, I. C., Zentek, J., and Vahjen, W. (2015). Effects of the probiotic Enterococcus faecium NCIMB 10415 on selected lactic acid bacteria and enterobacteria in co-culture. Benef. Microbes 6, 345-352. doi: 10.3920/BM2014.0052

Tian, L., Bruggeman, G., van Den Berg, M., Borewicz, K., Scheurink, A. J., Bruininx, E., et al. (2017). Effects of pectin on fermentation characteristics, carbohydrate utilization, and microbial community composition in the gastrointestinal tract of weaning pigs. Mol. Nutr. Food Res. 61, 1-10. doi: 10.1002/mnfr.201600186

Trompette, A., Gollwitzer, E. S., Yadava, K., Sichelstiel, A. K., Sprenger, N., Ngom-Bru, C., et al. (2014). Gut microbiota metabolism of dietary fiber influences allergic airway disease and hematopoiesis. Nat. Med. 20, 159-166. doi: $10.1038 / \mathrm{nm} .3444$

Tsai, T., Chien, H. F., Wang, T. H., Huang, C. T., Ker, Y. B., and Chen, C. T. (2011). Chitosan augments photodynamic inactivation of gram-positive and gram-negative bacteria. Antimicrob. Agents Chemother. 55, 1883-1890. doi: 10.1128/AAC.00550-10

Vijayaraghavan, P., Arun, A., Al-Dhabi, N., Vincent, S., Arasu, M., and Choi, K. (2016). Novel Bacillus subtilis IND19 cell factory for the simultaneous production of carboxy methyl cellulase and protease using cow dung substrate in solid-substrate fermentation. Biotechnol. Biofuels 22, 73. doi: 10.1186/s13068-016-0481-6

Yang, J. J., Niu, C. C, and Guo, X. H. (2015). Mixed culture models for predicting intestinal microbial interactions between Escherichia coli and Lactobacillus in the presence of probiotic Bacillus subtilis. Benef. Microbes 6, 871-877. doi: 10.3920/BM2015.0033

Conflict of Interest Statement: The authors declare that the research was conducted in the absence of any commercial or financial relationships that could be construed as a potential conflict of interest.

Copyright $\odot 2017$ Liu, Zhao, Guo, Lu, Geng, Levesque, Johnston, Wang, Liu, Zhang, $\mathrm{Ma}$, Qiao and Ma. This is an open-access article distributed under the terms of the Creative Commons Attribution License (CC BY). The use, distribution or reproduction in other forums is permitted, provided the original author(s) or licensor are credited and that the original publication in this journal is cited, in accordance with accepted academic practice. No use, distribution or reproduction is permitted which does not comply with these terms. 